\title{
Correlation of Neurologic Dysfunction with CT Findings in Early Acute Stroke
}

\author{
J.N. Scott, A.M. Buchan and R.J. Sevick
}

\begin{abstract}
Objective: To determine the frequency of early computed tomographic (CT) findings of ischemia and their relationship to symptom duration and neurologic dysfunction within 3 hours of ischemic stroke. Methods: The CT scans of 39 acute stroke patients were evaluated for signs of early ischemic change within 3 hours of symptom onset and without knowledge of the patient's neurologic deficit or results of a 24 hour follow-up post-thrombolysis CT. Early CT signs of acute ischemic change or thromboembolism were hypoattenuation of the insular ribbon, obscuration of the lentiform nucleus, cortical hypodensity/effacement, and hyperdense middle cerebral artery sign. Results: Signs of acute ischemic change were seen on the baseline scan in $25 / 39$ patients (64\%). Hypoattenuation of the insular ribbon was seen in 11 patients, obscuration of the lentiform nucleus in 13, cortical hypodensity/effacement in 13, and hyperdense middle cerebral artery sign in 7 . The prevalence of early ischemic signs was directly associated with increasing neurologic disability at the time of presentation. No clear relationship existed between symptom duration and the presence of CT signs. Conclusions: Evidence of cerebral ischemia is frequently seen on CT within 3 hours of symptom onset. The degree of neurologic disability correlates with $\mathrm{CT}$ signs of ischemia.
\end{abstract}

RÉSUMÉ: Corrélation entre la dysfonction neurologique et les observations au CT scan dans l'accident vas-
culaire cérébral en phase précoce. Objectif: II s'agit de déterminer la fréquence de manifestations précoces d'is-
chémie à la tomographie assistée par ordinateur (CT) et leur relation avec la durée de la symptomatologie et avec la
dysfonction neurologique, en dedans de trois heures d'un accident vasculaire cérébral ischémique (AVCI). Méthodes:
Nous avons examiné le CT scan de 39 patients présentant un AVCI pour détecter des signes précoces de changements
ischémiques en dedans de 3 heures du début des symptômes et sans connaître le déficit neurologique du patient ou les
résultats du CT fait 24 heures après la thrombolyse. Les signes précoces de changements ischémiques aigus au CT ou
de thrombo-embolie étaient une hypoatténuation du ruban insulaire, une obscuration du noyau lenticulaire, une hypo-
densité/un effacement cortical et le signe de l'artère cérébrale moyenne hyperdense. Résultats: Des signes de change-
ments ischémiques aigus ont été observés au scan initial chez 25 patients sur 39 (64\%). Une hypoatténuation du ruban
insulaire a été observée chez 11 patients, une obscuration du noyau lenticulaire chez 13 , une hypodentité/un efface-
ment cortical chez 13 et le signe de l'artère cérébrale moyenne hyperdense chez 7 . La prévalence de signes
ischémiques précoces était directement associée à un déficit neurologique progressif au moment où le patient a con-
sulté. Il n'existait pas de relation claire entre la durée des symptômes et la présence de signes au CT scan. Conclusions:
Des manifestations d'ischémie cérébrale sont observées fréquemment au CT scan en dedans de 3 heures du début des
symptômes. Le degré de dysfonction neurologique est en corrélation avec les signes d'ischémie observés au CT scan.

Can. J. Neurol. Sci. 1999; 26: 182-189

The primary role of CT scanning in patients with sudden onset of neurologic symptoms has been the detection of intracranial hemorrhage and to exclude disease processes that may mimic ischemia (e.g., tumor or subdural hematoma). However, the increasing use of thrombolytic therapy as treatment for acute stroke necessitates that stroke team members also recognize often subtle changes on CT scan which may represent early ischemic change.

Signs of acute cerebral ischemia or thromboembolism have been described by others and include hypoattenuation of the insular ribbon, ${ }^{1}$ obscuration of the lentiform nucleus, ${ }^{2}$ cortical hypodensity/effacement of other hemispheric territories, ${ }^{1-3}$ and the hyperdense middle cerebral artery (MCA) sign. . $^{4-6}$
The presence of CT signs of hemispheric brain ischemia may increase the risk of deterioration, with or without cerebral hemorrhage, after thrombolytic therapy. As such, clinical studies which have examined the benefit of thrombolytic therapy for stroke have emphasized that a short time from the ischemic event

From the Departments of Diagnostic Imaging (J.N.S., R.J.S.), Clinical Neurosciences (A.M.B., R.J.S.), and Anatomy (R.J.S.), Foothills Hospital, University of Calgary and the Alberta Stroke Program, Calgary, Alberta, Canada. R.J.S. is a member of the Seaman Family MR Research Centre. RECEIVED OCTOBER 22, 1998. ACCEPTED IN FINAL FORM MARCH 18, 1999. Reprint requests to: Dr. R.J. Sevick, Department of Diagnostic Imaging, Foothills Hospital, 1331 - 29 Street N.W., Calgary, Alberta, Canada T2N 4N2 
to treatment and the absence of any sign of brain injury on CT are important inclusion criteria. ${ }^{7,8}$

This clinical and radiological study was prompted after reviewing the CT scans of patients treated with recombinant tissue plasminogen activator (rt-PA) at our stroke center in which we found a higher frequency of acute ischemic signs than was originally expected. Our study is a practical extension of the application of rt-PA in acute ischemic stroke presenting within 3 hours of symptom onset, and evaluates the prevalence and distribution of early ischemic signs in a large patient population. The results of this analysis may aid members of the stroke team in developing acute management strategies.

\section{Materials and Methods}

Our regional stroke program serves a population of approximately 1.5 million. Patients who present at our institution early after suspected stroke are referred urgently to the acute stroke treatment team. Patients are evaluated by the stroke neurologist, and CT scan and appropriate laboratory studies are obtained before the patient is transferred to the acute stroke unit where a final decision regarding thrombolytic therapy is made. Patients are entered into an acute stroke database and all imaging studies are archived.

We reviewed the CT scans and medical records of 39 consecutive prospectively gathered patients between April 1, 1996 and December 21, 1997 who presented within 3 hours of onset of an acute ischemic neurologic deficit and were treated with intravenous rt-PA. The patients were required to have had an ischemic stroke with a clearly defined time of onset, a deficit measurable using the National Institute of Health Stroke Scale (NIHSS), and a baseline CT scan of the brain that showed no evidence of intracranial hemorrhage.

The patients had a neurological examination and were scored using the NIHSS immediately before rt-PA treatment. The NIHSS score is 0 for a healthy patient and 42 for a comatose patient with all scale items maximally impaired. Patients with minimal symptoms, rapidly improving clinical deficits, evidence of evolving infarction involving more than one-third of the compromised vascular territory, or other previously described exclusion criteria $^{7}$ were not considered candidates for thrombolytic therapy and were therefore not included in this study.

All patients had at least two CT scans, the first within 3 hours of symptom onset and the second CT approximately 24 hours post-acute management. All baseline CT scans were reviewed by two of the authors without knowledge of the patients' clinical symptoms or their time of onset, or of any findings on the subse- quent CT studies. In cases where the baseline scan was felt to be normal, the 24-hour study was then assessed for regions of acute ischemia which, in retrospect, could also be seen as abnormal on the initial study.

CT scans of the brain without intravenous contrast were performed on most patients using a Toshiba Xpress SX helical scanner with the remaining studies performed using a General Electric HiSpeed Advantage scanner. Images were acquired using contiguous axial 6-mm sections from the foramen magnum to the suprasellar region and $10-\mathrm{mm}$ contiguous slices through the remainder of the brain.

Signs of acute ischemic change, and the parent vessel territory of the ischemic region were documented as follows. Hypoattenuation of the insular ribbon was defined as loss of differential density between subcortical white and gray matter of the insular cortex. Obscuration of the lentiform nucleus was defined as a decrease in density involving the lentiform nucleus and loss of gray-white matter differentiation in this area. Cortical hypodensity/effacement was defined as an area of hypodensity within a cortical region other than the insula, or subtle mass effect with effacement of cortical sulci. Hyperdense MCA sign was defined as hyperdensity within the MCA that was greater than the contralateral MCA or any other intracranial vessel of similar size not attributable to calcification.

The data were expressed as mean \pm standard error of the mean (S.E.M.) and analyzed with either a two-sample (unpaired) t-test or non-parametric test for comparison of two means (MannWhitney Two Sample Test).

\section{Results}

A total of 39 patients were evaluated and $78 \mathrm{CT}$ scans were reviewed. There were 24 male and 15 female patients with a mean age of $68.3 \pm 2.2$ years (range, 24 to 95 ). At admission, all patients presented with severe hemispheric symptoms that scored between 4 and 24 points (mean, $13.8 \pm(0.85)$ on the NIHSS. As an example, a typical patient with an NIHSS score of 9 and right MCA occlusion might exhibit a complete left hemianopia, minor left facial paresis, left hemiparesis with minimal effort against gravity of both arm and leg, partial sensory loss, and mild dysarthria.

\section{CT Characteristics}

The baseline CT was performed at a mean time of $112 \pm 5$ mins (range, 53 to 160 mins) from symptom onset. The baseline CT scan was normal in $36 \%$ of the patients and showed at least one abnormality in $64 \%$ (Table 1). The second CT was performed approximately 24 hours post-thrombolytic therapy and was abnormal in $85 \%$ of patients. All patients who had an early

Table 1: Summary of Clinical Characteristics and Computed Tomographic Scan Results in 39 Patients.

\begin{tabular}{lrlrl}
\hline Radiographic Features & \multicolumn{2}{c}{ Baseline CT scan } & \multicolumn{2}{c}{ 24 Hour Follow-up CT Scan } \\
& \multicolumn{1}{c}{$\boldsymbol{n}(\boldsymbol{\%})$} & \multicolumn{2}{c}{$\boldsymbol{n}(\boldsymbol{\%})$} \\
\hline Hypoattenuation of the insular ribbon & 11 & $(28 \%)$ & 11 & $(28 \%)$ \\
Obscuration of the lentiform nucleus & 13 & $(33 \%)$ & 16 & $(41 \%)$ \\
Cortical hypodensity/effacement & 13 & $(33 \%)$ & 16 & $(41 \%)$ \\
Hyperdense MCA sign & 7 & $(18 \%)$ & 2 & $(5 \%)$ \\
No acute findings & 14 & $(36 \%)$ & 6 & $(15 \%)$ \\
Hemorrhage' & 0 & $(0 \%)$ & 12 & $(31 \%)$ \\
\hline
\end{tabular}

${ }^{1}$ Varies from petechial hemorrhage to lobar hematomas. 

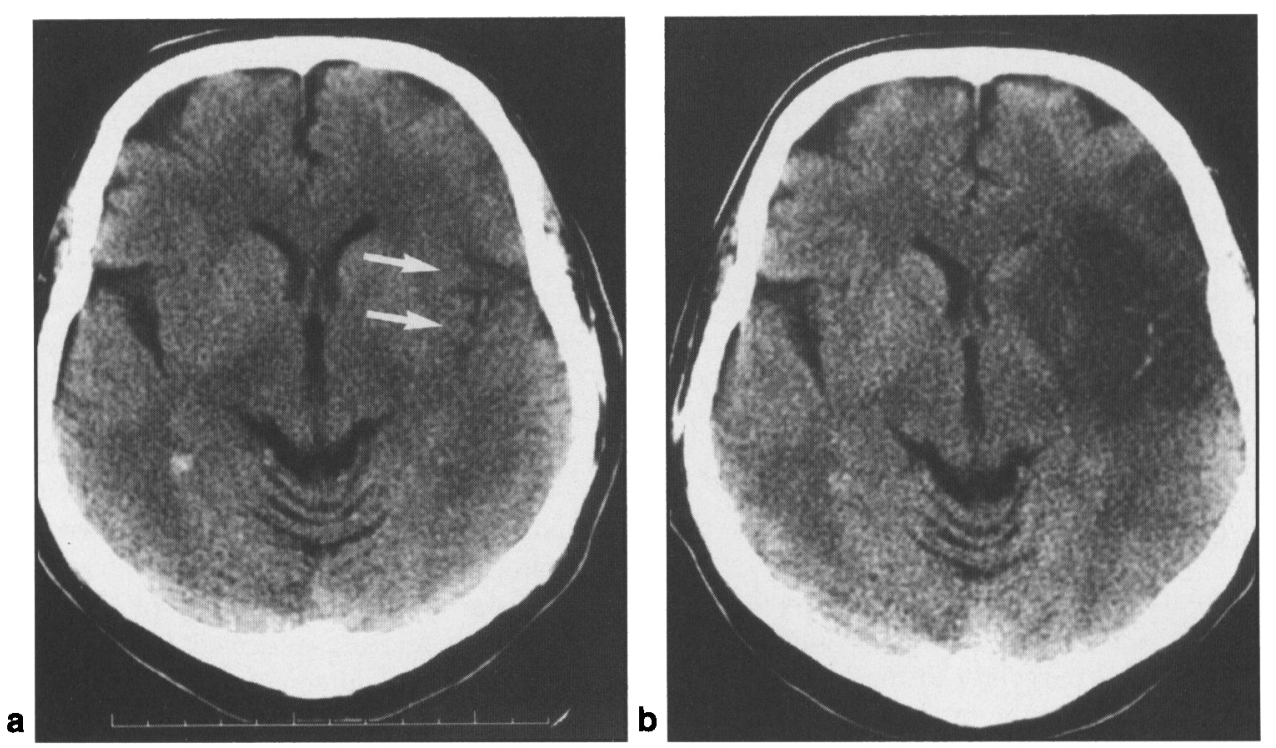

Figure 1: Hypoattenuation of the insular ribbon. (a) Hypodensity of the insular cortex with resultant decreased density of the affected gray matter (arrows). Note is also made of the low density of the adjacent temporal opercular cortex. (b) 24-hour follow-up CT scan shows large MCA territory infarction with hyperdense clot visible in Sylvian branches of the MCA.
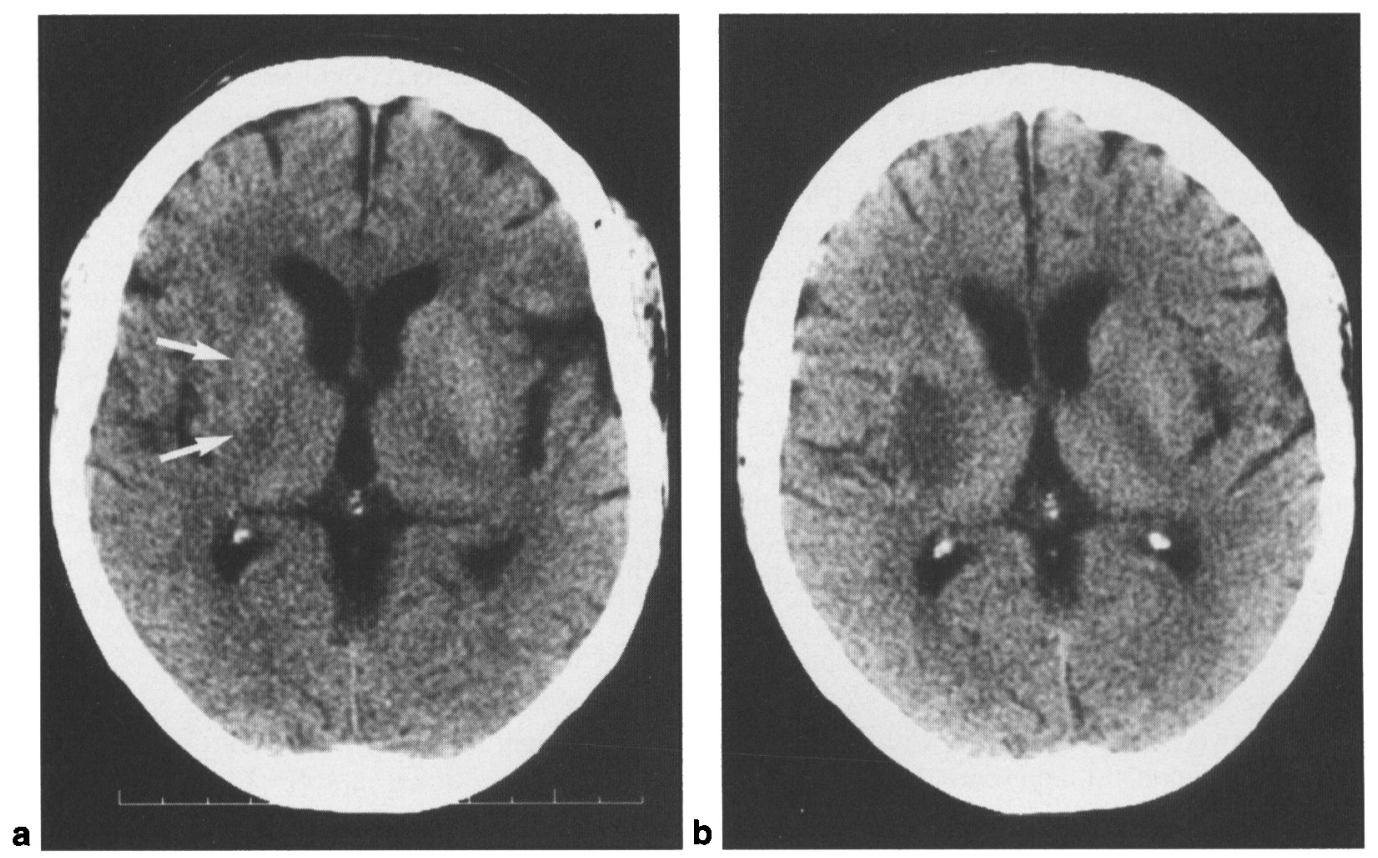

Figure 2: Obscuration of the lentiform nucleus. (a) Subtle decrease in density of the lentiform nucleus on the right (arrows) compared with its normal counterpart on the left. (b) Follow-up scan 24 hours later shows infarct localized to putamen and posterior limb of the internal capsule.

ischemic sign on their baseline scan had a corresponding positive sign on their follow-up study.

\section{Early Ischemic Signs}

The CT findings at varying intervals after the onset of symptoms are shown in Table 1 and in Figures 1 to 4 . On the baseline CT scan, hypoattenuation of the insular ribbon was identified in $11 / 39(28 \%)$ of patients, obscuration of the lentiform nucleus in $13(33 \%)$, cortical hypodensity/effacement in $13(33 \%)$, and hyperdense MCA sign in 7 (18\%). In five patients, subtle signs of acute ischemic change (hypoattenuation of the insular ribbon,
2; obscuration of the lentiform nucleus, 1; cortical hypodensity/effacement, 3) were seen retrospectively on the baseline CT after viewing the follow-up study. No early findings were noted on the baseline CT in $14(36 \%)$ patients. In addition to signs of early ischemic change, seven patients had evidence of old infarction on their baseline CT studies.

On the follow-up CT scan only six (15\%) studies were normal and detection of infarction was increased with hypoattenuation of the insular ribbon identified in $11 / 39(28 \%)$ of patients, obscuration of the lentiform nucleus in $16(41 \%)$, and cortical hypodensity/effacement in $16(41 \%)$. The hyperdense MCA sign was 

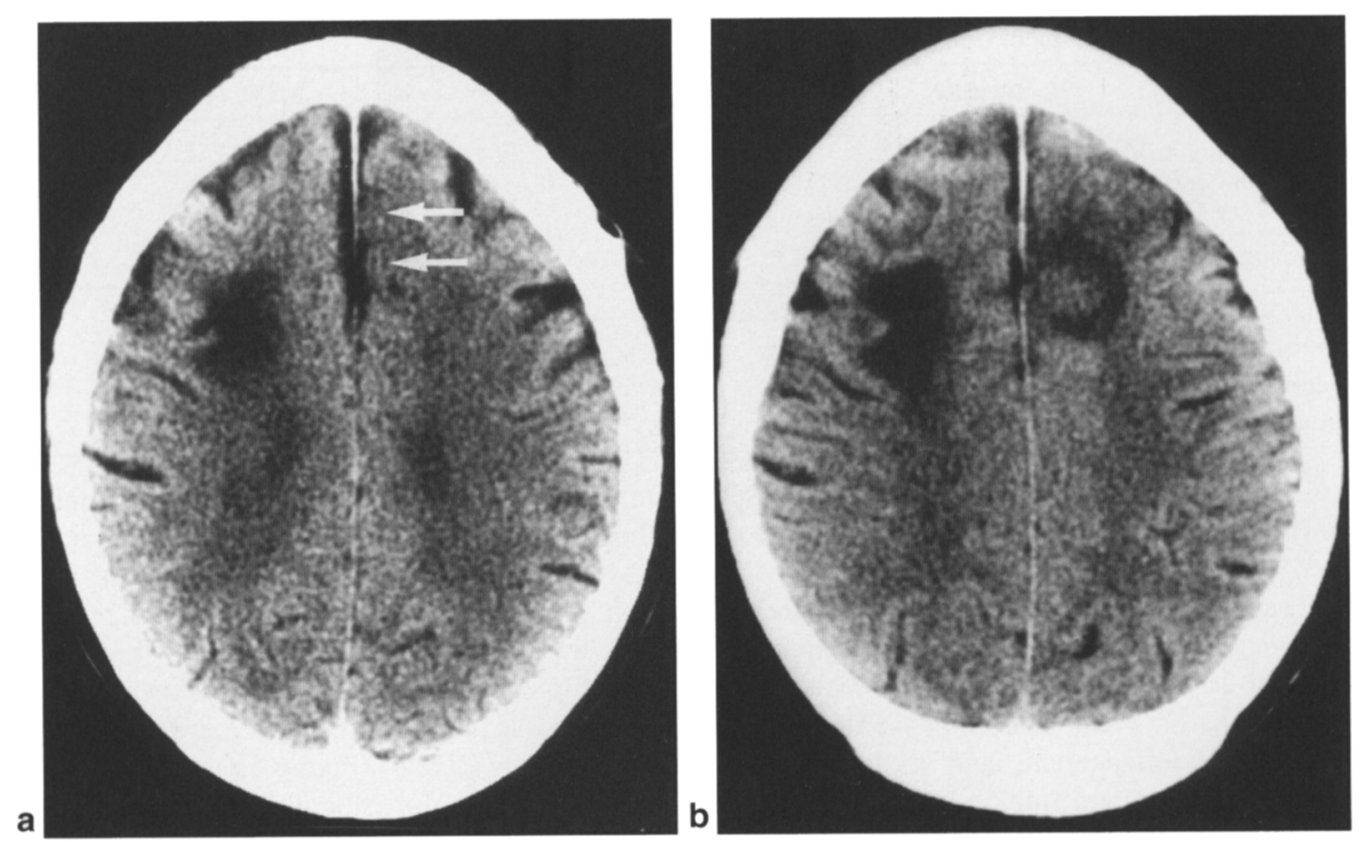

Figure 3: Cortical hypodensity/effacement in the ACA territory. (a) Decreased density of the affected cortical gray matter in the left medial frontal lobe (arrows). Incidental chronic right frontal infarct. (b) 24-hour follow-up CT scan shows small acute left frontal infarct.
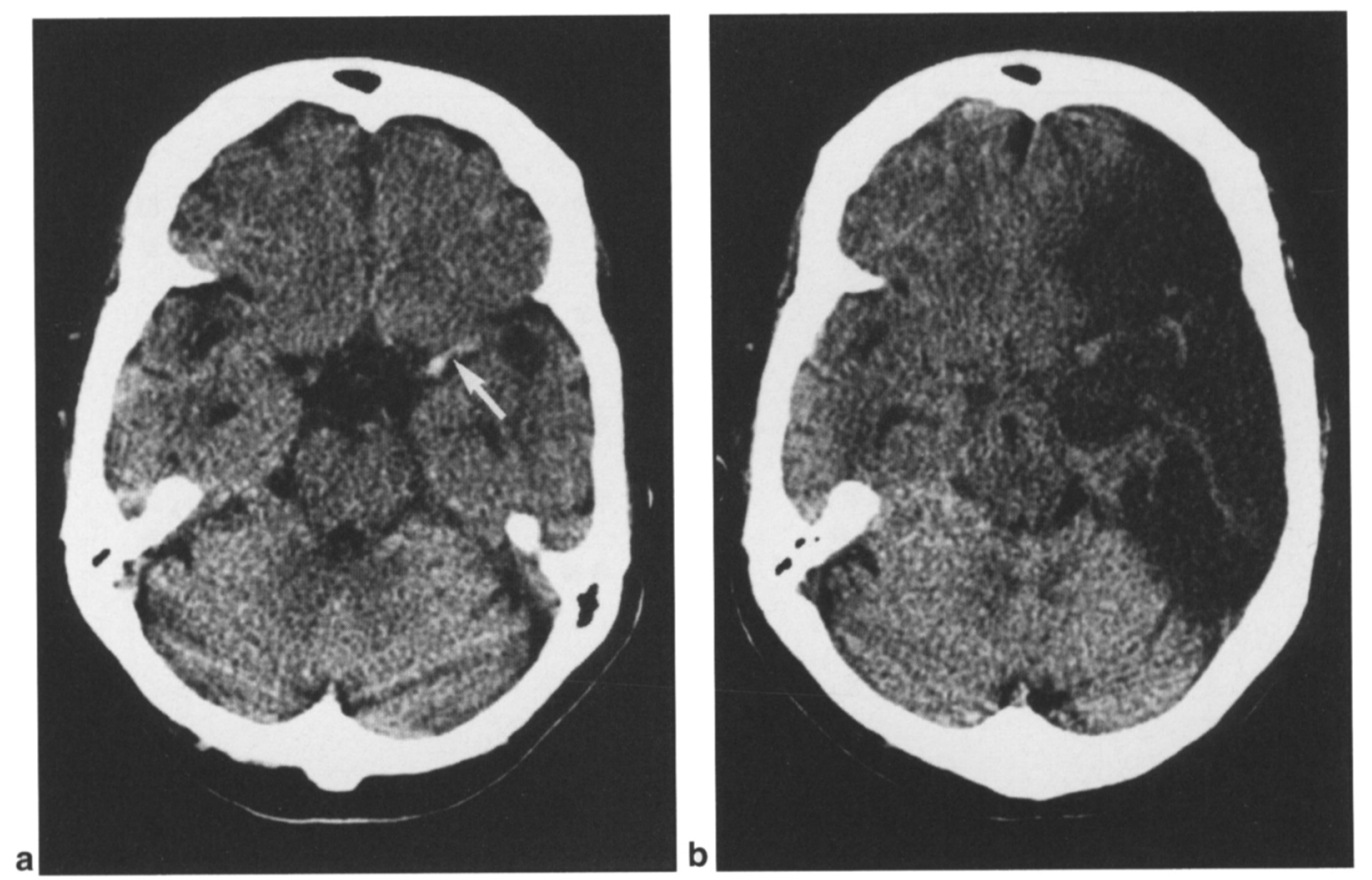

Figure 4: Hyperdense MCA sign. (a) Hyperdense clot is shown in the MI segment of the MCA (arrow). (b) 24-hour follow-up CT scan shows massive MCA territory infarction, the artery no longer appears hyperdense.

identified in 2 (5\%) patients (Table 1). In the 33 cases with positive ischemic changes, the infarcts involved the MCA vascular territory in 29 patients, the anterior cerebral artery (ACA) in two, and the posterior cerebral artery (PCA) in two. Secondary bleeding was present in $12 / 39(31 \%)$ cases and always occurred either within the infarction itself or the region corresponding to the presenting symptoms if the baseline study was normal. Every patient who had an early sign of infarction on their baseline CT had a corresponding infarction on the follow-up study except for three patients who had lobar hemorrhages.

\section{Time of Initial CT and Early Ischemic Signs}

The earliest CT scan that showed parenchymal hypoattenuation of any degree was obtained 53 minutes after the onset of symptoms. There was no significant difference in the initial clinical severity between patients with early ( $<90 \mathrm{mins}$; NIHSS 15.3 $\pm 1.86, n=9)$ and later (90-180 mins; NIHSS $13.3 \pm 0.95, n=$ 
Table 2: Correlation of Computed Tomographic Scan Results with Symptom Duration in 39 Patients.

\begin{tabular}{|c|c|c|c|c|c|}
\hline Symptom Duration & Insular $^{1}$ & Lentiform $^{2}$ & Cortex $^{3}$ & MCA clot ${ }^{4}$ & No Acute Findings \\
\hline $0-90$ mins $(n=9)$ & & & & & \\
\hline Mean $65 \pm 3.8$ mins (range, $53-76$ ) & $4(44 \%)$ & $2(22 \%)$ & $4(44 \%)$ & $3(33 \%)$ & $3(33 \%)$ \\
\hline Mean $127 \pm 3.1$ mins (range, $94-160$ ) & $7(23 \%)$ & $11(37 \%)$ & $9(30 \%)$ & $4(13 \%)$ & $11(37 \%)$ \\
\hline
\end{tabular}

'Insular, hypoattenuation of the insular ribbon (see Materials and Methods)

${ }^{2}$ Lentiform, obscuration of the lentiform nucleus

${ }^{3}$ Cortex, cortical hypodensity/effacement

${ }^{4} \mathrm{MCA}$ clot, hyperdense middle cerebral artery sign.

30) CT studies $(P=0.3275)$. The relative number of patients with normal CT studies was similar between the 0-90 min and 90-180 minute groups (Table 2). There was no clear association between the time at which the baseline CT was performed and signs indicating acute infarction or thromboembolism. A slightly greater proportion of patients who had their baseline CT scan within 90 minutes of symptom onset had hypoattenuation of the lentiform nucleus, cortical hypodensity/effacement, and a hyperdense MCA sign, whereas the most common finding between 90-180 minutes was obscuration of the lentiform nucleus.

\section{Severity of Neurologic Dysfunction and Early Ischemic Signs}

An increasing frequency of acute ischemic signs was associated with an increasing severity of neurological deficit at patient presentation (Figure 5). The NIHSS scores for patients with nor- mal CT scans (mean, $10.1 \pm 1.54$ ) were significantly lower than those for patients whose scans showed any ischemic finding (mean, $15.9 \pm 0.08)(P=0.0012)$ (Figure $5 \mathrm{~A})$; or hypoattenuation of the lentiform nucleus (mean, $16.2 \pm 1.41)(P=0.0092)$, obscuration of the lentiform nucleus (mean, 16.1 \pm 1.14$)(P=$ 0.0049 ), cortical hypodensity/effacement (mean, $15.9 \pm 1.25)(P$ $=0.0074$ ) (Figure 5B); or the hyperdense MCA sign (mean, 18.3 $\pm 1.54)(P=0.0034)$ (Figure 5B).

Furthermore, for patients with a NIHSS of 1 to 10 , no acute ischemic signs were detected in $9 / 10$ (90\%) cases (Table 3 ). A single patient had hypoattenuation of the insular ribbon, obscuration of the lentiform nucleus and cortical hypodensity/effacement. In contrast, only $3 / 17(18 \%)$ patients with an NIHSS of 11 to 16 , and $2 / 12(17 \%)$ with an NIHSS of 17 to 24 had normal CT scans.

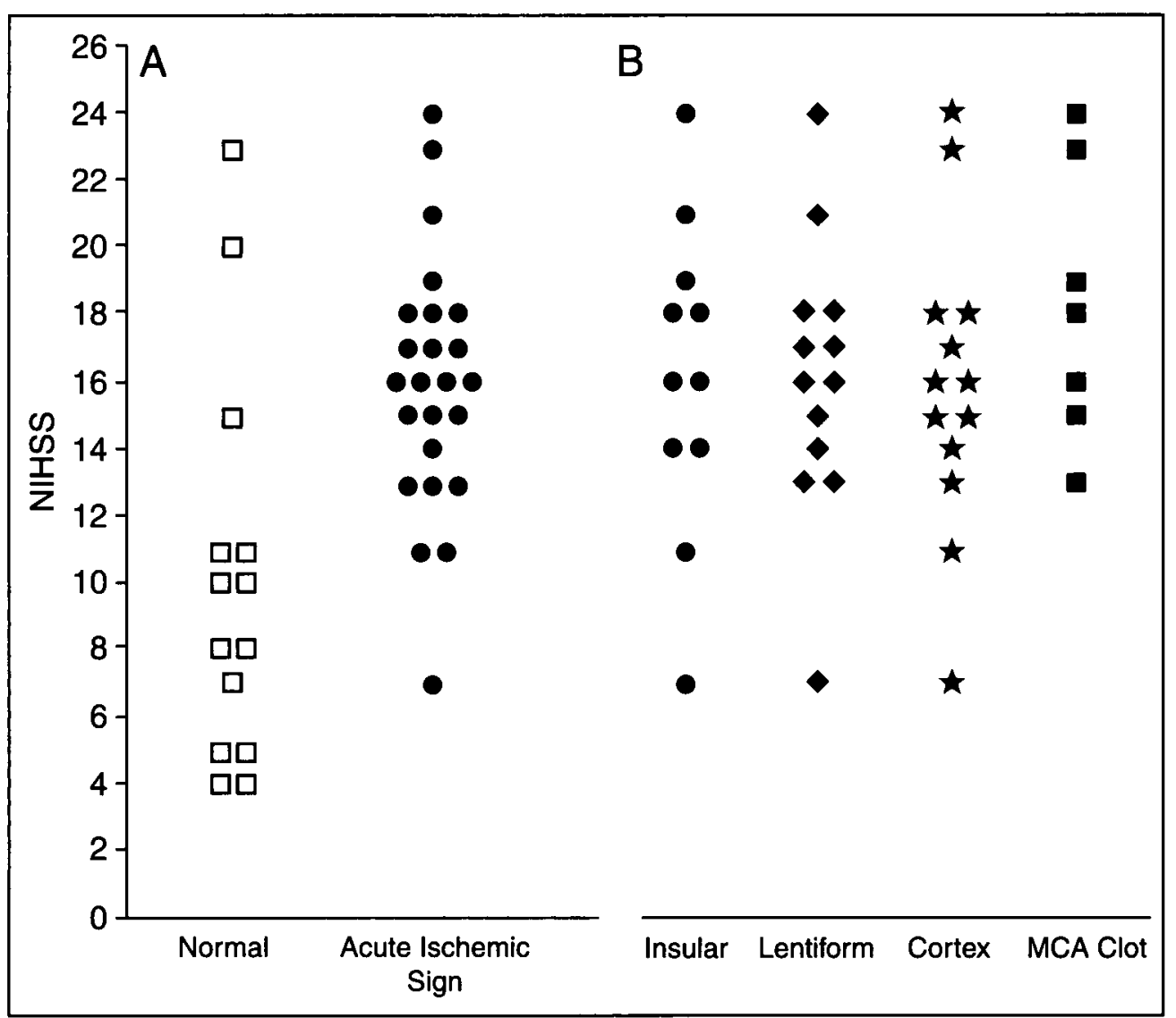

Figure 5: Correlation of NIHSS with CT scan results in 39 patients. (A) Comparison between normal and positive CT scans with any sign of acute ischemia or thromboembolism, and $(B)$ with the individual signs. Insular, hypoattenuation of the insular ribbon; Lentiform, obscuration of the lentiform nucleus; Cortex, cortical hypodensity/effacement; $M C A$ clot, hyperdense MCA sign. 
Table 3: Correlation of NIHSS ${ }^{1}$ with Computed Tomographic Scan Results in 39 Patients.

\begin{tabular}{|c|c|c|c|c|c|c|}
\hline NIHSS & Insular ${ }^{2}$ & Lentiform $^{3}$ & Cortex $^{4}$ & MCA clot ${ }^{5}$ & & Acute Findings \\
\hline Mean $6.8 \pm 0.71$ & $1(10 \%)$ & $0(0 \%)$ & $1(10 \%)$ & $1(10 \%)$ & 9 & $(90 \%)$ \\
\hline Mean $13.8 \pm 0.46$ & $5(29 \%)$ & $3(18 \%)$ & $6 \quad(35 \%)$ & $7(41 \%)$ & 3 & $(18 \%)$ \\
\hline $17-24(n=12)$ & & & & & & \\
\hline Mean $19.6 \pm 0.74$ & $5(42 \%)$ & $4(33 \%)$ & $6 \quad(50 \%)$ & $5(42 \%)$ & 2 & $(17 \%)$ \\
\hline
\end{tabular}

${ }^{1}$ NIHSS, National Institutes of Health Stroke Scale (see Materials and Methods)

${ }^{2}$ Insular, hypoattenuation of the insular ribbon

${ }^{3}$ Lentiform, obscuration of the lentiform nucleus

${ }^{4}$ Cortex, cortical hypodensity/effacement

${ }^{5} \mathrm{MCA}$ clot, hyperdense middle cerebral artery sign

\section{Discussion}

It has been previously reported that detection of ischemic changes on CT images within 6 hours of a stroke is poor. ${ }^{9-10}$ In fact, until recently, the general consensus has been to use CT scans following infarction to exclude hemorrhage, subdural hematoma, or mass rather than the demonstration of infarction. The earliest CT scanners initially gave the impression that infarction could not be seen prior to 24 hours after symptom onset, ${ }^{11,12}$ although an increasing number of reports have now described detection of several well defined signs of acute ischemic change. ${ }^{1 \cdot 6,13,14}$ We set out to confirm our impression that CT signs which indicate acute ischemia can be found much earlier and in a higher percentage of patients with acute stroke than was originally believed.

Early CT abnormalities were found in the majority of our patients $(64 \%)$ within 3 hours of symptom onset, which is similar to previous reports that vary from 40 to $80 \% \%^{1,2,15}$ depending upon the time that the CT scan was performed. In our series, individual signs of acute ischemic change were evident in $67 \%$ of patients whose baseline CT was performed within 90 minutes of symptom onset. Selection of patients from the literature who were scanned within the same time period yields a similar rate of detection between 38 and $75 \% .^{1,2,15}$ However, it is important to note that many additional patients within our study period were excluded from analysis because their ischemic changes were deemed too extensive for them to receive rt-PA. Therefore, our data likely underestimate the true frequency of early CT signs of ischemia within a general stroke population.

The importance of recognizing findings of early ischemia on CT scans is paramount for several reasons. The recognition of parenchymal hypodensity and/or effacement of sulci in more than one-third of the MCA territory is often used as an exclusion criteria for patients receiving intravenous thrombolytic therapy. ${ }^{8,15}$ In addition to increasing age and severity of clinical deficit, ${ }^{16}$ the risk of hemorrhagic transformation of the infarct following recanalization and reperfusion post-thrombolysis is thought to increase with enlarging size of the ischemic lesion on CT. ${ }^{17}$ Finally, assessment of early ischemic CT signs has been reported to help predict clinical response to thrombolytic therapy, with patients having smaller ischemic regions benefiting to a greater extent. ${ }^{15,18}$

Hypoattenuation of the insular ribbon, or decreased density of the affected gray matter at the lateral margin of the insula, was initially described by Truwit $^{1}$ as the most frequently observed sign of acute ischemia within the MCA territory. The blood supply to the insular region is provided mainly by the insular segment of the MCA and its claustral branches. In MCA occlusion distal to the lenticulostriate arteries, the insular ribbon becomes the region furthest from potential collateral flow from the anterior and posterior cerebral circulation and so it effectively becomes a watershed zone. In our series, hypoattenuation of the insular ribbon was frequently seen early after the onset of ischemia, even before 90 minutes, although its overall frequency was similar to other signs.

Obscuration of the lentiform nucleus has been described as a common sign of acute infarction, often being recognized as early as one hour after symptom onset. ${ }^{2}$ This deep MCA territory is also extremely sensitive to ischemia and is likely because the lenticulostriate arteries which supply it are end-arteries with no potential for collateral blood flow. In our series, obscuration of the lentiform nucleus was the most common sign overall and its presence correlated well with subsequent deep MCA infarction (positive predictive value $100 \%$ and negative predictive value $88 \%)$.

Hemispheric cortical hypoattenuation (other than insular cortex) and sulcal effacement occurred together in almost all cases in our series. Cortical hypodensity/effacement was found in $33 \%$ of our patients which is similar to previous reports that vary from 33 to $46 \%$ within 3 hours of symptom onset. ${ }^{1,2}$ However, in the present study and others, ${ }^{1,2,13}$ cortical hypoattenuation/effacement is a relatively infrequent early ischemic sign in isolation ( 0 to $17 \%$ ) which likely reflects, in part, the greater proportion of MCA versus non-MCA territory strokes. In our series, only 5/39 (13\%) patients had an isolated cortical hypodensity/effacement sign; four of which were within either the ACA or PCA vascular territories.

The hyperdense MCA sign is an indirect sign of ischemia corresponding to arterial occlusion that may be visualized on early noncontrast CT scans before an infarct becomes evident. While increased attenuation may in fact represent a thrombus within the MCA, adult patients occasionally manifest atherosclerotic calcifications that can simulate an acute thrombus, ${ }^{1}$ suggesting that the hyperdense MCA sign may not be a reliable indicator of vessel occlusion. ${ }^{19}$ The strict definition we used required that the hyperdensity within the MCA be greater than the contralateral MCA, or any other intracranial vessel of similar size not attributable to calcification, to be considered positive ${ }^{13,20}$ and likely reflects a more accurate incidence in the setting of acute stroke. 
Hyperdense MCA sign was present in only seven (18\%) of our patients while previous studies have described higher incidences, varying between 33 to $47 \% .^{1,21-23}$ However, several of these reports ${ }^{1,21,22}$ have excluded non-MCA territory strokes which could artifactually elevate its frequency when comparing to a general stroke population. The lysis of clot, due to endogenous thrombolytics, is a natural phenomenon which thrombolytic therapy is seeking to accelerate and this would account for only two of the hyperdense MCA signs persisting on the follow-up studies. 22.24 .25

Our results show a clear association between the number of early CT ischemic signs and initial clinical severity. 7,8,13,15,21,26 Increasing neurologic disability, as measured using the NIHSS, correlated closely with the presence of CT signs of ischemia in the setting of acute stroke. Furthermore, $90 \%$ of patients with a low NIHSS (0-10) had negative CT studies compared to only $17 \%$ in the more disabled groups. This relationship most likely reflects the individual's available collateral circulation, ${ }^{27}$ with those patients who have fewer collaterals experiencing more severe ischemia and developing increased neurologic impairment and early CT changes. In contrast, no significant relationship between symptom duration and the presence of ischemic signs in the acute setting was evident, ${ }^{15}$ with similar percentages of patients having negative CT studies in each of the two time periods examined.

Hemorrhagic transformation, symptomatic and asymptomatic, was observed in 12 of the 39 patients $(31 \%)$ on the $24-$ hour follow-up CT scans. This frequency is similar to previous reports of acute stroke patients treated with rt-PA. $^{7,8,28}$ Hemorrhagic transformation is a common accompaniment of embolic infarction even in patients not treated with thrombolytics and appears related to both the intensity of ischemia and delayed infarct extension, but not with the presence of early CT signs of ischemia. ${ }^{13,22,28}$ Previous natural history studies have reported similar incidences of hemorrhagic transformation, varying between $33-43 \%$ in acute stroke patients evaluated by either serial $\mathrm{CT}^{13,17,29}$ or autopsy studies. ${ }^{30}$ More recently, the detection of deoxyhemoglobin by gradient echo magnetic resonance sequences, or methemoglobin on T1-weighted sequences provides an exquisite look at trace amounts of blood and illustrates that petechial hemorrhagic transformation is more frequent than CT studies suggest. ${ }^{31,32}$ Different variables, including the use of anticoagulants, embolic versus atherosclerotic stroke, and time elapsed from stroke onset have all been postulated to increase the risk of hemorrhagic transformation in stroke patients.

This study was a retrospective review of a group of stroke patients and the reviewers were aware that the patients were subsequently treated with thrombolytic therapy. Scan interpretation by stroke team members in a prospective manner may yield a lower rate of detecting acute ischemic changes. However, clinical information about the site of cerebral ischemia would improve the observer's detection of CT signs. Knowledge of the follow-up scans led to subtle signs being detected in five additional patients and would not have altered treatment decisions.

In conclusion, the early diagnosis of ischemic infarction by $\mathrm{CT}$ depends on recognition of often subtle changes of reduced attenuation and slight mass effect. It is only through gaining further knowledge of the particular early CT changes, in collaboration with other studies that correlate long-term outcome with or without use of thrombolytics, that we can make informed initial decisions regarding use of thrombolysis for the individual patient. The present study reinforces that early ischemic changes are frequently present within the first few hours of cerebral infarction and, as a result, the CT scan is now more carefully examined at our center. The presence of early ischemic signs correlated more closely with the severity of the patient's neurologic dysfunction than the length of time from symptom onset in the acute setting. Recognition of early CT signs of ischemia should aid members of the stroke team in developing acute management strategies for their patients.

\section{Abbreviations}

$\mathrm{CT}$, computed tomography, MCA, middle cerebral artery, NIHSS, National Institute of Health Stroke Scale; rt-PA, recombinant tissue plasminogen activator.

\section{ACKNOWLEDGEMENTS}

We thank our patients and their families for participating in this study, and Peggy Kochanski and Marilyn Mora for their expert assistance. This study was supported in part by the Heart and Stroke Foundation of Alberta and an infrastructural grant from the Alberta Stroke Program.

\section{REFERENCES}

1. Truwit CL, Barkovich AJ, Gean-Marton A, Hibri N, Norman D. Loss of the insular ribbon: another early CT sign of acute middle cerebral artery infarction. Radiology 1990; 176: 801-806.

2. Tomura $\mathrm{N}$, Uemura $\mathrm{K}$, Inugami A, et al. Early CT findings in cerebral infarction: obscuration of the lentiform nucleus. Radiology 1988; 168: 463-467.

3. Bozzao L, Bastianello S, Fantozzi LM, et al. Correlation of angiographic and sequential CT findings in patients with evolving cerebral infarction. Am J Neuroradiol 1989a; 10: 1215-1222.

4. Gács G, Fox AJ, Barnett HJM, Vinuela F. CT visualization of intracranial arterial thromboembolism. Stroke 1983; 14: 756-762.

5. Pressman BD, Tourje EJ, Thompson JR. An early CT sign of ischemic infarction: increased density in a cerebral artery. Am J Neuroradiol 1987; 8: 645-648.

6. Schuierer G, Huk W. The unilateral hyperdense middle cerebral artery: an early CT-sign of embolism or thrombosis. Neuroradiology 1988; 30: 120-122.

7. The National Institute of Neurological Disorders and Stroke rt-PA Stroke Study Group. Tissue plasminogen activator for acute ischemic stroke. N Engl J Med 1995; 333: 1581-1587.

8. Hacke W, Kaste M, Fieschi C for the ECASS Study Group. Intravenous thrombolysis with recombinant tissue plasminogen activator for acute hemispheric stroke: the European Cooperative Acute Stroke Study (ECASS). JAMA 1995; 274: 1017-1059.

9. Inoue $\mathrm{Y}$, Takemoto K, Miyamoto $\mathrm{T}$, et al. Sequential computed tomography scans in acute cerebral infarction. Radiology 1980; 135: $655-662$.

10. Weingarten $\mathrm{K}$. Computed tomography of cerebral infarction. Neuroimaging Clin N Am 1992; 2: 409-419.

11. Campbell JK, Houser DW, Stevens JC, et al. Computed tomography and radionuclide imaging in the evaluation of ischemic stroke. Radiology 1978; 126: 695-702.

12. Kingsley DPE, Radue EW, DuBoulay EPGH. Evaluation of computed tomography in vascular lesions of the vertebrobasilar territory. J Neurol Neurosurg Psychiatry 1980; 43: 193-197.

13. Moulin T, Cattin F, Crépin-Leblond T, et al. Early CT signs in acute middle cerebral artery infarction: predictive value for subsequent infarct locations and outcome. Neurology 1996; 47: 366-375.

14. von Kummer R, Nolte PN, Schnittger $H$, Thron A, Ringelstein EB. Detectability of cerebral hemisphere ischaemic infarcts by $\mathrm{CT}$ within $6 \mathrm{~h}$ of stroke. Neuroradiology 1996; 38: 31-33. 
15. von Kummer R, Allen KA, Holle R, et al. Acute stroke: usefulness of early CT findings before thrombolytic therapy. Radiology 1997; 205: 327-333.

16. Larrue V, von Kummer R, del Zoppo G, Bluhmki E. Hemorrhagic transformation in acute ischemic stroke. Potential contributing factors in the European cooperative acute stroke study. Stroke 1997; 28: 957-960.

17. Toni D, Fiorelli M, Bastianello S, et al. Hemorrhagic transformation of brain infarction: predictability in the first 5 hours from stroke onset and influence on clinical outcome. Neurology 1996; 46: 341-345.

18. Trouillas P, Nighoghossian N, Getenet J-G et al. Open trial of intravenous tissue plasminogen activator in acute carotid territory stroke. Correlations of outcome with clinical and radiological data. Stroke 1996; 27: 882-890.

19. Rauch RA, Bazan III C, Larsson E-M, Jinkins JR. Hyperdense middle cerebral arteries identified on CT as a false sign of vascular occlusion. Am J Neuroradiol 1993; 14: 669-673.

20. Granström, P: CT visualization of thrombus in cerebral artery. J Comput Assist Tomogr 1986; 10: 541-542.

21. Tomsick T, Brott T, Barsan W, et al. Prognostic value of the hyperdense middle cerebral artery sign and stroke scale score before ultraearly thrombolytic therapy. Am J Neuroradiol 1996; 17: 79-85.

22. von Kummer R, Meyding-Lamadé U, Forsting M, et al. Sensitivity and prognostic value of early $\mathrm{CT}$ in occlusion of the middle cerebral artery trunk. Am J Neuroradiol 1994; 15: 9-15.

23. Tomsick T, Brott T, Barsan W, et al. Thrombus localization with emergency cerebral CT. Am J Neuroradiol 1992; 13: 257-263.
24. Leys D, Pruvo JP, Godefroy O, Rondepierre P, Leclerc X. Prevalence and significance of hyperdense middle cerebral artery in acute stroke. Stroke 1992; 23: 317-324.

25. Bastianello S, Pierallini A, Colonnese $\mathrm{C}$, et al. Hyperdense middle cerebral artery CT sign. Comparison with angiography in the acute phase of ischemic supratentorial infarction. Neuroradiology 1991; 33: 207-211.

26. Brott T, Marler JR, Olinger CP, et al. Measurements of acute cerebral infarction: lesion size by computed tomography. Stroke 1989; 20: 871-875.

27. Bozzao L, Fantozzi LM, Bastianello S, Bozzao A, Fieschi C. Early collateral blood supply and late parenchymal brain damage in patients with middle cerebral artery occlusion. Stroke 1989b; 20: 735-740.

28. Wolpert SM, Bruckmann H, Greenlee R, et al. Neuroradiologic evaluation of patients with acute stroke treated with recombinant tissue plasminogen activator. Am J Neuroradiol 1993; 14: 3-13.

29. Hornig CR, Dorndorf W, Agnoli AL. Hemorrhagic cerebral infarction: a prospective study. Stroke 1986; 17: 179-185.

30. Lodder J, Krijne-Kubat B, Broekman J. Cerebral hemorrhagic infarction at autopsy: cardiac embolic cause and the relationship to the cause of death. Stroke 1986; 17: 626-629.

31. Bryan RN, Levy LM, Whitlow WD, et al. Diagnosis of acute cerebral infarction: comparison of $\mathrm{CT}$ and MR imaging. Am J Neuroradiol 1991; 12: 611-620.

32. Hornig CR, Bauer T, Simon C, Trittmacher S, Dorndorf W Hemorrhagic transformation in cardioembolic cerebral infarction. Stroke 1993; 24: 465-468. 